\title{
Impact of Viral PCR Positive Nasal Swabs (Non Covid-19) on Outcomes Following Cardiac Surgery
}

\author{
Virginia Kathleen Cox $^{1} \cdot$ Diane Hersey $^{1} \cdot$ Melissa Valentine $^{1} \cdot$ Keyana Richardson $^{1} \cdot$ Lanette Johnson $^{1}$. \\ Mark Galantowicz ${ }^{1}$ Janet M. Simsic ${ }^{1}$ (I)
}

Received: 14 January 2021 / Accepted: 5 May 2021 / Published online: 13 May 2021

(c) The Author(s), under exclusive licence to Springer Science+Business Media, LLC, part of Springer Nature 2021

\begin{abstract}
Viral bronchiolitis is a relative contraindication to elective pediatric cardiac surgery. Nasopharyngeal swab utilizing polymerase chain reaction (PCR) screening for viruses known to cause bronchiolitis are commonly available. The objective of this study was to evaluate clinical outcomes in patients with nasopharyngeal viral PCR positive findings at the time of cardiac surgery. Retrospective review from January 2013 to May 2019 for patients with virus detected by PCR on nasopharyngeal swabs at the time of cardiac surgery. Single ventricle and two ventricle patients were compared to control group of age and procedure matched patients viral negative at the time of surgery. Outcome measures included OR extubation, reintubation, hospital length of stay, and mortality. For two ventricle patients $(n=81$; control group $=165)$, there was no statistical difference in any outcome variable (OR extubation $74 \%$ vs $72 \% ; p=0.9$; reintubation $9 \%$ vs $11 \%$ vs; $p=0.7$; hospital length of stay 5 days (1-46) vs 4 days (2-131); $p=0.4$; mortality 2 vs $1 ; p=0.3)$. For single ventricle patients, there was no statistical difference in any outcome variable (OR extubation $81 \%$ vs $76 \% ; p=0.6$; reintubation $14 \%$ vs $21 \%$ vs; $p=0.5$; hospital length of stay 9.5 days (3-116) vs 15 days $(2-241) ; p=0.1$; mortality 0 vs $3 ;(p=0.6))$. PCR is a sensitive test that fails to predict which patients will proceed to have a clinically significant infection. Viral bronchiolitis remains a relative risk factor for cardiac surgery; presence of detectable virus via nasopharyngeal swab with limited clinical symptoms may not be a contraindication to cardiac surgery.
\end{abstract}

Keywords Congenital heart disease $\cdot$ Cardiac surgery $\cdot$ Viral bronchiolitis

\section{Introduction}

Viral bronchiolitis is a relative contraindication to elective pediatric cardiac surgery [1-3]. Human rhinovirus is responsible for increased morbidity in patients following cardiac surgery [1]. RSV infection has been associated with respiratory failure and need for mechanical ventilation, as well as, increased morbidity and mortality in infants with congenital heart disease; especially those with pulmonary hypertension and cyanosis [2]. Congenital heart disease, T21 and prematurity are independent risk factors for severe bronchiolitis $[4,5]$. It is common for cardiac surgery to be delayed for 4-6 weeks in a patient with suspected viral bronchiolitis regardless of symptoms.
It has become common practice to screen for viruses prior to cardiac surgery. However, screening is usually based on patient diagnosis or risk factors, symptoms or history of sick contact as it is not practical to test all patients. Nasopharyngeal swab utilizing polymerase chain reaction (PCR) screening for viruses known to cause bronchiolitis are commonly available [6]. PCR testing requires viral DNA; it does not require viral antigen. Therefore, the tests are sometimes positive in asymptomatic children [7]. It is a very sensitive test for the detection of virus, but positive test may not identify or predict symptomatic illness [8]. The objective of this retrospective study was to evaluate clinical outcomes in patients with nasopharyngeal viral PCR positive findings at the time of cardiac surgery.

Janet M. Simsic

janet.simsic@ nationwidechildrens.org

1 The Heart Center at Nationwide Children's Hospital, 700

Children's Drive, Columbus, OH 43205, USA 


\section{Methods}

This retrospective study was approved by the Institutional Review Board at our institution with waiver of informed consent. Nationwide Children's Hospital is an academic, nonprofit, freestanding children's hospital located in Columbus, Ohio. The cardiothoracic intensive care unit is a 20-bed unit with over 400 cardiac surgeries per year. All cardiothoracic surgical patients undergoing repair or palliation of congenital heart disease are admitted to the cardiothoracic intensive care unit for postoperative management. The top five most common admission diagnoses are postoperative ventricular septal defect repair, hybrid palliation stage I, hybrid palliation comprehensive stage II, pulmonary valve replacement and Fontan procedure. Approximately $10 \%$ of admissions are newborns, defined as $<30$ days of age.

All single and two ventricle patients who had viruses detected by PCR on nasopharyngeal swabs at the time of cardiac surgery from January 2013 to May 2019 were retrospectively reviewed. During this timeframe, patients were screened with nasopharyngeal swabs for viral bronchiolitis if they exhibited symptoms, regardless of the severity of symptoms, or if there was a history of sick contact at home or school. All single ventricle patients and those with T21 or pulmonary hypertension were also screened regardless of symptoms or sick contacts as we considered these patients to potentially be higher risk of poor outcome if undergoing cardiac surgery while viral positive. Surgery was postponed if patients exhibited significant symptoms including respiratory distress or fever, regardless of PCR results. Therefore, the patients who were PCR viral positive that proceeded with cardiac surgery were largely asymptomatic or mildly symptomatic. The PCR viral positive patients who proceeded with cardiac surgery without postponement were compared to a control group of age and procedure matched patients that were viral negative at the time of surgery or had no clinical indication to warrant respiratory viral testing. Cases and control subjects were matched 1:2. Outcome measures included extubation in the operating room, reintubation within $24 \mathrm{~h}$ after a planned extubation, hospital length of stay, and mortality.

Viral bronchiolitis was documented by nasopharyngeal swabs for 12 respiratory viruses (rhinovirus, influenza $\mathrm{A}$ [subtypes H1 and H3], influenza B, respiratory syncytial virus [subtypes $A$ and $B$ ], parainfluenza virus [types 1, 2, and 3], human metapneumovirus, and adenovirus) using a multiplex PCR platform (Luminex Molecular Diagnostics, Austin, TX, USA) throughout the study period. Sensitivity of the PCR respiratory viral panel fast assay compared to gold standard PCR was 79\%; specificity was 99\% [9].

\section{Statistical Analysis}

Continuous variables were summarized using mean and standard deviation as well as median and range. Categorical variables were summarized as number and percentage. Continuous data were analyzed using a two-sample $t$ test. Categorical data were analyzed using a two-tailed Fischer's exact test. Statistical significance was defined a $p<0.05$.

\section{Results}

\section{Two Ventricle Patients}

There were 81 two ventricle patients in the viral positive group who were compared to 165 two ventricle patients in the control viral negative group. There was no statistical difference between the viral positive group and the viral negative group in any variable as demonstrated in Table 1 . The study and control patients were similar in age, weight, and surgical procedure as classified by STAT category. The most common virus isolated on PCR in the two ventricle patients was human rhinovirus/enterovirus $N=61$ (75\%) followed by adenovirus $N=12(15 \%)$. The rest of the viral breakdown was as follows: coronavirus non Covid-19 $N=6$; parainfluenza $N=5$ and RSV $N=4$. Seven patients tested positive for more than one virus.

Table 1 Two ventricle patients

\begin{tabular}{llll}
\hline & FARVAPP positive & $\begin{array}{l}\text { FARVAPP } \\
\text { negative } \\
\text { control }\end{array}$ & $p$ value \\
\hline$N=$ & 81 & 165 & \\
$\begin{array}{l}\text { Age (years) aver- } \\
\text { age } \pm \text { stdev }\end{array}$ & $4.1 \pm 8.5$ & $3.5 \pm 6.3$ & \\
Median (range) & $0.9(0.02-45)$ & $0.8(0.01-51)$ & 0.2 \\
Weight (kg) aver- & $16 \pm 21$ & $15 \pm 20$ & 0.7 \\
age \pm stdev & & & \\
Median (range) & $8(3.4-110)$ & $8(2.1-132)$ & \\
Syndrome & $23(28 \%)$ & $45(27 \%)$ & 0.9 \\
STAT & & & \\
1 & $31(38 \%)$ & $70(42 \%)$ & 0.6 \\
2 & $23(28 \%)$ & $34(21 \%)$ & 0.2 \\
3 & $13(16 \%)$ & $32(19 \%)$ & 0.6 \\
4 & $14(17 \%)$ & $29(18 \%)$ & 1 \\
Extubation in OR & $60(74 \%)$ & $119(72 \%)$ & 0.9 \\
Reintubation & $7(9 \%)$ & $19(11 \%)$ & 0.7 \\
Hospital LOS (days) & $8 \pm 8.1$ & $12 \pm 21$ & 0.4 \\
Median (range) & $5(1-46)$ & $4(2-131)$ & \\
Mortality & 2 & 1 & 0.3 \\
\hline
\end{tabular}


Three two ventricle patients had hospital length of stay $>30$ days. The first was a 7-year-old female from Trinidad (adenovirus positive) with atrioventricular canal defect and trisomy 21 who presented for atrioventricular canal repair, pulmonary artery debanding, pulmonary artery patch reconstruction, mitral valve replacement. Her prolonged hospital length of stay (35 days) was related to postoperative cardiac arrest, heart block, and chylous effusion. The second was a 5-month-old female with anomalous coronary artery (parainfluenza positive) from the pulmonary artery with decreased ventricular function who underwent repair followed by left ventricular assist device. Her prolonged hospital length of stay (41 days) was not related to her viral bronchiolitis. The third patient was a 5-month-old female with pulmonary stenosis (human rhinovirus/enterovirus positive) who underwent pulmonary valvotomy, transannular patch, and monocuspid pulmonary valve placement. Her postoperative course was complicated by prolonged requirement for noninvasive positive pressure and oxygen which may have been related to her viral bronchiolitis (length of stay 46 days).

Fourteen viral positive patients with T21 who underwent cardiac surgery were compared to 24 viral negative patients with T21. Importantly, there was no statistical difference in any outcome variable in this high-risk population (OR extubation 7 of 14 vs 18 of $24 ; p=0.4$; hospital length of stay 4.5 days (3-24) vs 5 days (3-24); $p=0.4$ ).

Table 2 Single ventricle patients

\begin{tabular}{llll}
\hline & FARVAPP positive & $\begin{array}{l}\text { FARVAPP } \\
\text { negative } \\
\text { control }\end{array}$ & $p$ value \\
\hline$N=$ & 36 & 108 & \\
Age (years) aver- & $1.4 \pm 1.56$ & $1.4 \pm 1.6$ & 0.8 \\
age \pm stdev & & & \\
Median (range) & $0.6(0.15-6.5)$ & $0.6(0.05-7.3)$ & \\
Weight (kg) aver- & $9.1 \pm 3.7$ & $8.8 \pm 4$ & 0.5 \\
age \pm stdev & & & \\
Median (range) & $8.1(3.45-20)$ & $7.5(2.2-21)$ & \\
STAT score & & & \\
STAT 1 & 0 & 0 & 1 \\
STAT 2 & 24 & 71 & \\
STAT 3 & 0 & 0 & 0.7 \\
STAT 4 & 4 & 10 & 1 \\
STAT 5 & 8 & 27 & 0.6 \\
Extubated in OR & $29(81 \%)$ & $82(76 \%)$ & 0.5 \\
Reintubation & $5(14 \%)$ & $23(21 \%)$ & 0.1 \\
Hospital LOS (days) & $22 \pm 27$ & $21 \pm 34$ & $12(2-244)$ \\
Median (range) & $9.5(3-116)$ & 3 & 0.6 \\
Mortality & 0 & & \\
\hline
\end{tabular}

\section{Single Ventricle Patients}

There were 36 single ventricle patients in the viral positive group and 108 single ventricle patients in the viral negative control group. There was no statistical difference between the viral positive group and the viral negative group in any variable as demonstrated in Table 2 . The study and control patients were also similar in age, weight, and surgical procedure as classified by STAT category. The most common virus isolated on PCR in the single ventricle patients was human rhinovirus/enterovirus $N=27$ (75\%) followed by adenovirus $N=6(17 \%) . \%)$. The rest of the viral breakdown was as follows: parainfluenza $N=3$; RSV $N=3$; human metapneumovirus $N=1$; and coronavirus non Covid-19 $N=1$. Five patients tested positive for more than one virus.

Four single ventricle patients had hospital length of stay $>30$ days. The first was a 9-month-old male with double outlet right ventricle, malposed great vessels, subvalvar and valvar pulmonary stenosis, chromosomal abnormality (16P12.2 deletion) (human metapneumovirus positive) who underwent bidirectional Glenn and main pulmonary artery banding. Postoperative course was complicated by chylous effusion, hydrocephalus requiring ventriculoperitoneal shunt, and abdominal wall cellulitis not related to viral bronchiolitis (length of stay 116 days). The second was a 2-year-old male with hypoplastic left heart syndrome (human rhinovirus/enterovirus positive) who underwent Fontan procedure. His length of stay (53 days) was related to prolonged chest tube drainage not viral bronchiolitis. The third was an 8-month-old female with Turner Syndrome and unbalanced atrioventricular canal defect (human rhinovirus/ enterovirus positive) who underwent comprehensive stage II palliation. Postoperative course was complicated by systemic desaturation requiring inhaled nitric oxide which may have been related to viral bronchiolitis (length of stay 63 days). The fourth was a 2-month-old female with dextrocardia, tricuspid atresia, transposition of the great arteries, ventricular septal defect (parainfluenza positive) admitted with mesenteric ischemia who underwent pulmonary banding. Post-op course complicated by recurrence of hematochezia and pneumatosis not related to viral bronchiolitis (length of stay 31 days).

\section{Discussion}

Historically, viral bronchiolitis has been considered a relative contraindication to elective pediatric cardiac surgery. Children with congenital heart disease are independent risk factors for severe bronchiolitis $[4,5]$. We sought to challenge dogma and review outcomes of patients who were viral positive at the time of cardiac surgery in the current era. We found that preoperative identification of viral 
bronchiolitis by PCR alone did not predict increased morbidity or mortality.

RSV infection has been associated with respiratory failure and need for mechanical ventilation, as well as, increased morbidity and mortality in infants with congenital heart disease; especially those with pulmonary hypertension and cyanosis [2]. The introduction of palivizumab prophylaxis for patients with congenital heart disease was followed by a decrease in morbidity and mortality in this patient population [10]. Delgado-Corcoran et al. reported increase resource utilization and prolonged postoperative recovery after pediatric cardiac surgery in patients who were human rhinovirus positive and symptomatic [1]. Patients with PCR positive human rhinovirus had a ten-fold increase in utilization of noninvasive positive pressure ventilation following extubation; a 12-fold increase in reintubation; and an increased utilization of pulmonary medications. This led to longer cardiac intensive care unit and hospital lengths of stay control patients [1]. Spaeder et al. also found that patients with viral respiratory illness had longer postoperative ICU and hospital lengths of stay when compared to matched controls, $p<0.01$ [3]. In these studies, the patients were both viral positive and symptomatic. Symptomatic viral bronchiolitis remains a relative contraindication for cardiac surgery.

However, our study examined patients who were viral positive but only mildly symptomatic or asymptomatic. Like our study, Delgado-Corcoran et al. showed no statistical difference in clinical outcomes including intensive care and hospital lengths of stay, intubation time, and respiratory complications comparing preoperative positive PCR asymptomatic infants undergoing cardiac surgery to those with a negative PCR undergoing cardiac surgery [11]. This challenges the dogma that all pediatrics patients who are viral positive at the time of cardiac surgery should be postponed for 4-6 weeks.

The positive test with lack of symptoms may be explained by the following. PCR testing requires viral DNA; it does not require viral antigen. Therefore, the tests are sometimes positive in asymptomatic children [7]. It is a very sensitive test for the detection of virus, but positive test may not identify or predict symptomatic illness [8]. We believe that testing remains warranted in patients with mild symptoms, sick contacts or high-risk population as identification of potential viral bronchiolitis may result in an overall reduction of risk. Benefit of testing may decrease the in hospital viral transmission as these patients can be isolated with appropriate precautions for patient caregivers (gowns, gloves and mask).

\section{Limitations}

Limitations to this study include single institution and the retrospective nature of the study. Not all patients received PCR testing for viruses prior to cardiac surgery. Only those considered in a high-risk group (single ventricle, T21 or pulmonary hypertension), patients with symptoms or sick contacts were tested preoperatively. The definition of symptoms and sick contacts and therefore the decision to test was at the discretion of the cardiothoracic nurse practitioner performing preadmission testing. The decision to postpone surgery for patients who were viral positive or those with significant symptoms was at the discretion of the surgeon, cardiologist and anesthesiologist based on the needs of the individual patient. This study did not capture those patients postponed secondary to significant viral symptoms. The effect of poly viral infections could not be analyzed in this study cohort due to small sample size.

\section{Conclusion}

PCR is a sensitive test that fails to predict which patients will proceed to have a clinically significant infection. Preoperative identification of viral bronchiolitis by PCR alone did not predict increased morbidity or mortality. Symptomatic viral bronchiolitis remains a relative risk factor for cardiac surgery; presence of detectable virus via nasopharyngeal swab with limited clinical symptoms may not be a contraindication to cardiac surgery.

Author Contributions All authors have made substantial contributions to the conception or design of the work; or the acquisition, analysis, or interpretation of data; drafted the work or revised it critically for important intellectual content; approved the version to be published; and agree to be accountable for all aspects of the work in ensuring that questions related to the accuracy or integrity of any part of the work are appropriately investigated and resolved. Study concept and design: VKC, DH, MV, KR, LJ, MG, JMS. Acquisition, analysis or interpretation of data: VKC, DH, MV, KR, LJ, JMS. Drafting of the manuscript: VKC, DH, JMS. Statistical analysis: JMS.

Funding None.

Data Availability Data and material are available upon request to the corresponding author.

Code Availability Not applicable.

\section{Declarations}

Conflict of interest None of the authors have any conflicts of interest. None of the authors have any financial disclosures. 
Ethical Approval This retrospective study was approved by the Institutional Review Board at our institution with waiver of informed consent.

Consent for Publication All authors give their consent for publication to Pediatric Cardiology.

\section{References}

1. Delgado-Corcoran C, Witte MK, Ampofo K, Castillo R, Bodily S, Bratton SL (2014) The impact of human rhinovirus infection in pediatric patients undergoing cardiac surgery. Pediatr Cardiol 35:1387-1394. https://doi.org/10.1007/s00246-014-0941-3

2. Khongphatthanayothin A, Wong PC, Samara Y, Newth CJ, Wells WJ, Starnes VA, Chang AC (1999) The impact of respiratory syncytial virus infection on surgery for congenital heart disease: postoperative course and complications. Crit Care Med 27:1974-1981

3. Spaeder MC, Carson KA, Vricella LA, Alejo DE, Holmes KW (2011) Impact of the viral respiratory season on postoperative outcomes on children undergoing cardiac surgery. Pediatr Cardiol 32:801-806

4. Bloemers BL, van Furth AM, Weijerman ME, Gemke RJ, Broers CJ, van den Ende K, Kimpen JL, Strengers JL, Bont LJ (2007) Down syndrome: a novel risk factor for respiratory syncytial virus bronchiolitis: a prospective birth-cohort study. Pediatrics 120:e1076-e1081

5. Garcia CG, Bhore R, Soriano-Fallas A, Trost M, Chason R, Ramilo O, Mejias A (2010) Risk factors in children hospitalized with RSV bronchiolitis vs non-RSV bronchiolitis. Pediatrics 126:e1453-e1460

6. Brittain-Long R, Nord S, Olofsson S, Westin J, Anderson LM, Lindh M (2008) Multiplex real-time PCR for detection of respiratory tract infections. J Clin Virol 41:53-56

7. Jartti T, Jartti L, Peltola V, Waris M, Ruuskanen O (2008) Identification of respiratory viruses in asymptomatic subjects: asymptomatic respiratory virus infections. Pediatr Infect Dis J 27:1103-1107

8. Mahony JB, Petrich A, Smieja M (2011) Molecular diagnosis of respiratory virus infections. Crit Rev Sci 48(5-6):217-249

9. Gadsby NJ, Hardie A, Claas ECJ, Templeton KE (2010) Comparison of the Luminex respiratory panel fast assay with in-house real-time PCR for respiratory viral infection diagnosis. J Clin Microbiol 48(6):2213-2216

10. Resch B, Michel-Behnke I (2013) Respiratory syncytial virus infections in infants and children with congenital heart disease: update on the evidence of prevention with palivizumab. Curr Opin Cardiol 28:85-91

11. Delgado-Corcoran C, Blaschke AJ, Ou Z, Presson AP, Burch PT, Pribble CG, Menon SC (2019) Respiratory testing and hospital outcomes in asymptomatic infants undergoing heart surgery. Pediatr Cardiol 40:339-348. https://doi.org/10.1007/ s00246-018-1994-5

Publisher's Note Springer Nature remains neutral with regard to jurisdictional claims in published maps and institutional affiliations. 